\title{
QUASI-INTERPOLATORY SPLINES BASED ON SCHOENBERG POINTS
}

\author{
V. DEMICHELIS
}

\begin{abstract}
By using the Schoenberg points as quasi-interpolatory points, we achieve both generality and economy in contrast to previous sets, which achieve either generality or economy, but not both. The price we pay is a more complicated theory and weaker error bounds, although the order of convergence is unchanged. Applications to numerical integration are given and numerical examples show that the accuracy achieved, using the Schoenberg points, is comparable to that using other sets.
\end{abstract}

\section{INTRODUCTION}

In several recent papers [1-5] Dagnino et al. studied the application of quasiinterpolatory (QI) splines to numerical integration. This class of QI splines, introduced in [6], can be defined, starting with a positive integer $p \geq 2$, the order of the spline space, a partition

$$
Y_{N}: y_{0 N}:=a<y_{1 N}<\cdots<y_{N N}<y_{N+1, N}:=b,
$$

and a set of positive integers $\left\{d_{j N} ; j=0, \ldots, N+1\right\}$, where $d_{0 N}=d_{N+1, N}=p$ and $d_{j N}<p, j=1, \ldots, N$. Now we set $n:=\sum_{j=0}^{N} d_{j N}$ and write $\Pi_{n}$ for the nondecreasing sequence $\left\{x_{i n} ; i=1, \ldots, n+p\right\}$ obtained from $Y_{N}$ by repeating $y_{j N}$ exactly $d_{j N}$ times, $j=0, \ldots, N+1$. The norm $H_{n}$ of the set $\Pi_{n}$ is defined by

$$
H_{n}:=\max _{1 \leq i \leq n+p-1}\left(x_{i+1, n}-x_{i n}\right) .
$$

We shall assume throughout this paper that

$$
H_{n} \rightarrow 0 \quad \text { as } \quad n \rightarrow \infty .
$$

Let $\mathbf{P}_{p}$ be the set of polynomials of order $p$, or degree $\leq p-1$; we define the spline space $\mathbf{S}_{p, \pi_{n}}$ by

$\mathbf{S}_{p, \pi_{n}}:=\left\{g:\left.g\right|_{\left(y_{j N}, y_{j+1, N}\right)} \in \mathbf{P}_{p}, j=0, \ldots, N\right.$ and $g^{(i)}\left(y_{j N}^{+}\right)=g^{(i)}\left(y_{j N}^{-}\right), i=$ $\left.0,1, \ldots, p-d_{j N}-1 ; j=1, \ldots, N\right\}$.

Thus $\mathbf{S}_{p, \pi_{n}}$ is the class of polynomial splines of order $p$, with knots and boundary points $y_{j N}, j=0, \ldots, N+1$, of multiplicity $d_{j N}$. Since $d_{j N}<p, j=1, \ldots, N$, our splines will be continuous in $[a, b]$.

Received by the editor June 10, 1994.

1991 Mathematics Subject Classification. Primary 65D30, 65D05.

Work supported by "Ministero dell'Università e della Ricerca Scientifica e Tecnologica" and "Consiglio Nazionale delle Ricerche" of Italy. 
We say that the sequence of partitions $\left\{Y_{N}\right\}$ is locally uniform (LU) if there exists a constant $A \geq 1$ such that

$$
\left(y_{i+1, N}-y_{i N}\right) /\left(y_{j+1, N}-y_{j N}\right) \leq A,
$$

for all $0 \leq i, j \leq N$ with $|i-j|=1$ and for all $N$. We shall say that a sequence of spline spaces $\left\{\mathbf{S}_{p, \pi_{n}}\right\}$ is LU if the sequence of underlying partitions $\left\{Y_{N}\right\}$ is LU.

We consider in $\mathbf{S}_{p, \pi_{n}}$ the approximating splines $Q_{n} f$ of the form [6]

$$
\left(Q_{n} f\right)(x):=\sum_{i=1}^{n}\left(\sum_{j=1}^{l} \alpha_{i j}\left[\tau_{i 1}, \ldots, \tau_{i j}\right] f\right) N_{i n}^{p}(x),
$$

where

$$
N_{i n}^{p}(x):=\left(x_{i+p, n}-x_{i n}\right)\left[x_{i n}, \ldots, x_{i+p, n}\right] G_{p}(\cdot ; x), \quad i=1, \ldots, n,
$$

with

$$
G_{p}(t ; x):=(t-x)_{+}^{p-1} .
$$

The $N_{i n}^{p}$ are the normalized B-splines of order $p$ forming a basis for $\mathbf{S}_{p, \pi_{n}}$, $\left[z_{0}, \ldots, z_{p}\right] h$ is the $p$ th divided difference based on the points $z_{0}, \ldots, z_{p}, 1 \leq l \leq p$; the sets $\left\{\tau_{i 1}, \ldots, \tau_{i l} ; i=1, \ldots, n\right\}$ can be chosen in a suitable way and the $\alpha_{i j}$, defined below, are such that $Q_{n} g=g$ for all $g \in \mathbf{P}_{l}$ [6]. We assume that $\tau_{i j} \neq \tau_{i k}$ for $j \neq k$, so that the divided differences involve only function values. Under this assumption, $Q_{n} f$ can be expressed by [1]

$$
\left(Q_{n} f\right)(x):=\sum_{i=1}^{n} N_{i n}^{p}(x) \sum_{j=1}^{l} v_{i j} f\left(\tau_{i j}\right),
$$

where

$$
v_{i j}:=\sum_{\mu=j}^{l} \frac{\alpha_{i \mu}}{\prod_{\substack{s=1 \\ s \neq j}}^{\mu}\left(\tau_{i j}-\tau_{i s}\right)} .
$$

We consider now the following quantities [6]:

$$
E_{r s}^{n}(x):= \begin{cases}D^{r}\left(f-Q_{n} f\right)(x), & 0 \leq r<s, \\ D^{r} Q_{n} f(x), & s \leq r<p .\end{cases}
$$

We are interested in the different classes of QI points $\tau_{i j}$ such that, if $f \in$ $C^{s-1}[a, b]$ with $1 \leq s \leq l \leq p$, then for $0 \leq r<s$

$$
\left\|D^{r}\left(f-Q_{n} f\right)\right\|_{\infty} \leq C_{r} H_{n}^{s-r-1} \omega\left(D^{s-1} f ; H_{n} ;[a, b]\right),
$$

where, for any $g \in C(I)$,

$$
\omega(g ; \Delta ; I):=\max _{\substack{x, x+h \in I \\ 0<h \leq \Delta}}|f(x+h)-f(x)|
$$

and where $\|h\|_{\infty}:=\max _{a \leq x \leq b}|h(x)|$. 
When for all $1 \leq i \leq n$

$$
\tau_{i j} \in\left[x_{i n}, x_{i+p, n}\right], \quad j=1, \ldots, l,
$$

bounds for $\left|E_{r s}^{n}(x)\right|$ are given in [6, Theorems 5.2 and 5.3]. Using these bounds, one can prove that (6) holds if we choose the $\tau_{i j}$ as the following set :

$$
T_{1}: \tau_{i j}:=x_{i n}+(j-1)\left[\left(x_{i+p, n}-x_{i n}\right) /(l-1)\right], \quad j=1, \ldots, l, i=1, \ldots, n .
$$

This choice is suggested in [6] and used in [1, 2]. A second choice, suggested in [4], is

$$
T_{2}: \tau_{i j}:=x_{i n}+\left(j-\frac{1}{2}\right)\left[\left(x_{i+p, n}-x_{i n}\right) / l\right], \quad j=1, \ldots, l, i=1, \ldots, n .
$$

For both sets $T_{1}$ and $T_{2}$ it is proved in [4] that (6), with $r=0$, holds for an arbitrary sequence of spline spaces $\left\{\mathbf{S}_{p, \pi_{n}}\right\}$, while, if $0<r<s$, the sequence of spline spaces has to be LU in order that (6) holds.

The parameter sets $T_{1}$ and $T_{2}$ give great flexibility in the definition of the spline space $\mathbf{S}_{p, \pi_{n}}$; multiple knots can be used and, for $r=0$, there is no restriction in the distance between distinct knots. However, to construct our approximation $Q_{n} f$, we need up to $n(l-1)-p+2$ evaluations of $f$ for $T_{1}$, and $n l$ for $T_{2}$.

We can reduce the number of evaluations of $f$ when $d_{j N}=1, j=1, \ldots, N$, by choosing the $\tau_{i j}$ as one of the following sets:

$$
\left.\begin{array}{l}
T_{3}: \tau_{i j}:=x_{i+j-1, n} \\
T_{4}: \tau_{i j}:=x_{i+j, n}
\end{array}\right\}, \quad j=1, \ldots, l, i=p, \ldots, n+1-p,
$$

in which case it makes sense to set $l=p$.

The sets $T_{3}$ and $T_{4}$ are suggested in [3]; the $\tau_{i j}$ are chosen here to be a subset of the set of knots $K_{i}:=\left\{x_{i n}, \ldots, x_{i+p, n}\right\}$ except near the endpoints where the knots are not all distinct, in which case the $\tau_{i j}$ can be given by $T_{1}$ for $i=1, n$, while for $i=2, \ldots, p-1$ and $i=n-p+2, \ldots, n-1$ the $\tau_{i j}$ are chosen to include the distinct knots in $K_{i}$ augmented by some of the points $\tau_{1 j}$ or $\tau_{n j}$ as the case may be. A more symmetric set, suggested in [4], is

$$
T_{5}: \tau_{i j}=\left(x_{i+j-1, n}+x_{i+j, n}\right) / 2, \quad j=1, \ldots, l, i=p, \ldots, n+1-p
$$

and $\tau_{i j}$ as in the definition of $T_{3}$ and $T_{4}$ for the remaining values of $i$.

For the sets $T_{3}-T_{5}$ it is proved in $[3,4]$ that $(6)$ holds if the sequence of spline spaces $\left\{\mathbf{S}_{p, \pi_{n}}\right\}$ is LU.

The number of function evaluations is $n+p-2$ for $T_{3}, T_{4}$, and $n+p+1$ for $T_{5}$. In general, the sets $T_{3}-T_{5}$ contain much fewer points, and hence it is more economical to use them. However, they require that all interior knots in the spline be simple, whereas using $T_{1}$ or $T_{2}$, we can place multiple knots anywhere we wish.

In order to achieve both the flexibility of $T_{1}$ and $T_{2}$ in the choice of the spline space and the economy of computation of $T_{3}-T_{5}$, we suggest the following set of parameters:

$$
T_{6}:\left\{\begin{array}{l}
\tau_{i 1}:=\zeta_{i}, \quad i=1, \ldots, n, \\
\tau_{i 2}:=\zeta_{i-1}, \tau_{i 3}:=\zeta_{i+1}, \ldots, \tau_{i l}:=\zeta_{g_{i}(l)}, \quad i=[l / 2]+1, \ldots, n-[l / 2],
\end{array}\right.
$$


where, for all real $x,[x]$ denotes the greatest integer less than or equal to $x$,

$$
g_{i}(l):= \begin{cases}i-[l / 2] & \text { if } l \text { is even, } \\ i+[l / 2] & \text { if } l \text { is odd }\end{cases}
$$

and $\zeta_{i}:=\left(x_{i+1, n}+\cdots+x_{i+p-1, n}\right) /(p-1), i=1, \ldots, n$, is the $i$ th Schoenberg point.

Near the endpoints, for $i=1, n$, the $\tau_{i \nu}$ with $\nu=2, \ldots, l$ are the Schoenberg points, respectively to the right of $\zeta_{1}$ if $i=1$ and to the left of $\zeta_{n}$ if $i=n$. For $i=2, \ldots,[l / 2](i=n-[l / 2]+1, \ldots, n-1)$, the $\tau_{i \nu}$ are defined as in $T_{6}$ for $\nu=1, \ldots, j$ with $j$ such that $\tau_{i j}=\zeta_{1}\left(\tau_{i j}=\zeta_{n}\right)$, whereas for $\nu=j+1, \ldots, l$, the $\tau_{i \nu}$ are respectively the Schoenberg points to the right of $\tau_{i, j-1}$ (to the left of $\left.\tau_{i, j-1}\right)$.

For $l=2$ the choice $T_{6}$ leads to the Schoenberg variation-diminishing spline since, when $\tau_{i 1}=\zeta_{i}$, then $\alpha_{i 2}=0, i=1, \ldots, n$ (see [6]).

Using $T_{6}$ to determine $Q_{n} f$, we need only $n$ evaluations of $f$. Moreover, we can place multiple knots anywhere in $[a, b]$.

In $\S 2$ we will prove that (6) holds if the sequence of spline spaces $\left\{\mathbf{S}_{p, \pi_{n}}\right\}$ is LU. Moreover, we will give a uniform bound for the derivatives $D^{r} Q_{n} f$, with $s \leq r<p$. In order to achieve this result, we will modify slightly the error estimates proved in [6], since the set of parameters $T_{6}$ does not satisfy the conditions (7). In $\S 3$ we will apply sequences of splines $\left\{Q_{n} f\right\}$ based on $T_{6}$ to problems in numerical integration, including product quadrature of Riemann integrable functions and Cauchy principal value $(\mathrm{CPV})$ integrals. We will also give some numerical examples.

\section{ERror bounds For QI SPLINES BASEd on Schoenberg POINTS}

In this section we give an estimate for $\left|E_{r s}(x)\right|$, defined by (5), with $Q_{n} f$ defined by (1) with parameters $T_{6}$. We base our procedure on the results of [6]. To simplify the notation in this section and in the next, we denote the knots by $x_{i}$ instead of $x_{i n}$.

First we need the following result proved in [6, Lemma 4.1].

Lemma 1. Suppose $Q_{n}$ is defined on a class of functions containing $\mathbf{P}_{l}$, and suppose $Q_{n}$ reproduces $\mathbf{P}_{l}$. Then, for any polynomial $g \in \mathbf{P}_{s}$ and any $f$ such that $D^{r} f$ exists, $0 \leq r<s \leq l \leq p$, there holds

$$
E_{r s}^{n}(x)= \begin{cases}D^{r}\left(R-Q_{n} R\right)(x), & 0 \leq r<s, \\ D^{r} Q_{n} R(x), & s \leq r<p,\end{cases}
$$

where $R(x):=f(x)-g(x)$.

For a fixed $a \leq t \leq b$, let $m$ be such that $t \in J_{m}:=\left[x_{m}, x_{m+1}\right)$. We write $I_{i \nu}$ for the smallest closed interval containing $\left\{\tau_{i j} ; j=1, \ldots, \nu\right\}$ and $I_{m}$ for the smallest closed interval containing $J_{m}$ and $\bigcup_{i=m+1-p}^{m} I_{i l}$. For the set of parameters $T_{6}$,

$$
I_{i j} \subseteq\left[x_{\bar{l}(i)}, x_{\bar{r}(i)}\right],
$$

for $j=1, \ldots, l$, where

$$
\bar{l}(i):= \begin{cases}p, & i=1, \ldots,[p / 2], \\ i-[p / 2]+1, & i=[p / 2]+1, \ldots, n-[p / 2], \\ n+2-p, & i=n+1-[p / 2], \ldots, n,\end{cases}
$$


and

$$
\bar{r}(i):= \begin{cases}2 p-1, & i=1, \ldots,[p / 2], \\ i+[p / 2]+p-1, & i=[p / 2]+1, \ldots, n-[p / 2], \\ n+p-1, & i=n+1-[p / 2], \ldots, n .\end{cases}
$$

Moreover, for $p \leq m \leq n$,

$$
I_{m} \subseteq\left[x_{l(m)}, x_{r(m)}\right]
$$

where

$$
l(m):=\bar{l}(m+1-p)
$$

and

$$
r(m):=\bar{r}(m) .
$$

We define now, for $f \in C^{s-1}\left(I_{m}\right)$,

$$
R(x):=f(x)-\sum_{i=0}^{s-1} \frac{f^{(i)}(t)}{i !}(x-t)^{i} .
$$

If $R(x)$ is defined by (14), so that $g(x)$ is the Taylor expansion of $f$ at $t$, then $R(x)$ and its first $s-1$ derivatives are 0 at $t$. Hence, to give a bound for $E_{r s}^{n}(t)$, it is only necessary to estimate $D^{r} Q_{n} R(t)$ [6].

By [6, Lemma 4.3], and since $\tau_{i 1}:=\zeta_{i}$ for all $i$, the coefficients $\alpha_{i j}$ in (1) can be defined by

$$
\alpha_{i j}:=\left\{\begin{array}{l}
0, \quad j=2, \\
{[(p-j) ! /(p-1) !] \sum\left(x_{\nu_{1}}-\tau_{i 1}\right) \cdots\left(x_{\nu_{j-1}}-\tau_{i, j-1}\right), \quad j=1,3, \ldots, l,}
\end{array}\right.
$$

where the sum is taken over all choices of distinct $\nu_{1}, \ldots, \nu_{j-1}$ from $i+1, \ldots, i+p-1$. This is a sum of $(p-1) ! /(p-j)$ ! terms.

We recall that $0<N_{i n}^{p}(x) \leq 1$ for $x \in\left(x_{i}, x_{i+p}\right)$ and $N_{i n}^{p}(x)=0$ otherwise, except that $N_{1 n}^{p}(a)=N_{n n}^{p}(b)=1$ [7]. Now, setting $\lambda_{i j} g:=\left[\tau_{i 1}, \ldots, \tau_{i j}\right] g$, we have for $t \in J_{m}$

$$
Q_{n} f(t)=\sum_{i=m+1-p}^{m} \sum_{\substack{j=1 \\ j \neq 2}}^{l} \alpha_{i j} \lambda_{i j} f N_{i n}^{p}(t)
$$

since all other terms in the sum (1) are zero, and

$$
D^{r} Q_{n} f(t)=\sum_{i=m+1-p}^{m} \sum_{\substack{j=1 \\ j \neq 2}}^{l} \alpha_{i j} \lambda_{i j} f D^{r} N_{i n}^{p}(t)
$$

From Lemma 1, $R(x)$ defined as in (14) satisfies

$$
\left|E_{r s}^{n}(t)\right|=\left|D^{r} Q_{n} R(t)\right| \leq \sum_{i=m+1-p}^{m}\left|D^{r} N_{i n}^{p}(t)\right| \cdot \sum_{\substack{j=1 \\ j \neq 2}}^{l}\left|\alpha_{i j}\right|\left|\lambda_{i j} R\right| .
$$

To give a bound for $\left|E_{r s}^{n}(t)\right|$, we need the following lemma [6, Lemma 2.1]: 
Lemma 2. Let $N_{i n}^{p}(x)$ be defined as in (2). Suppose $x \in J_{m}$ and $i \leq m<i+p$. Fix $0<r<p$. If $x=x_{m}$, suppose also that $x_{m}$ is of multiplicity at most $p-r-1$. Then the rth derivative $D^{r} N_{i n}^{p}(x)$ exists, and

$$
\left|D^{r} N_{i n}^{p}(x)\right| \leq \frac{\Gamma_{p r}}{\delta_{i m, p-1} \cdots \delta_{i m, p-r}},
$$

where, for $j=p-r, \ldots, p-1$, we define $\delta_{i m j}$ as the minimum of $x_{\nu+j}-x_{\nu}$ over $\nu$ such that $x_{i} \leq x_{\nu} \leq x<x_{\nu+j} \leq x_{i+p}$, and where

$$
\Gamma_{p r}:=\frac{(p-1) !}{(p-r-1) !}\left(\begin{array}{c}
r \\
{[r / 2]}
\end{array}\right) \text {. }
$$

By using (15) and (16) we state the following lemma [6, Lemma 4.4]:

Lemma 3. Suppose $Q_{n}$ reproduces polynomials $\mathbf{P}_{l}$, and $\alpha_{i j}$ are as in (15). Then, given $t \in J_{m}$ and $R$ as in Lemma 1, we have

$$
\left|D^{r} Q_{n} R(t)\right| \leq p \Gamma_{p r} \cdot \max _{m+1-p \leq i \leq m} \sum_{\substack{j=1 \\ j \neq 2}}^{l}\left|\lambda_{i j} R\right| A_{i j},
$$

where

$$
A_{i j}:=\left\{\begin{array}{l}
0, \quad j=2, \\
\max _{\substack{i+1 \leq \nu_{1} \ldots \nu_{j-1} \leq i+p-1 \\
\nu_{\mu} \text { distinct }}} \frac{\left|x_{\nu_{1}}-\tau_{i 1}\right| \cdots\left|x_{\nu_{j-1}}-\tau_{i, j-1}\right|}{\delta_{i m, p-1} \cdots \delta_{i m, p-r}}, \quad j=1,3, \ldots, l .
\end{array}\right.
$$

We need to introduce parameters describing the spacings of the $\tau_{i j}$ and the knots. For each integer $1 \leq \nu \leq l-1$ let

$$
\sigma_{i j \nu}:=\min _{1 \leq \mu \leq j-\nu}\left(\tau_{i, \mu+\nu}^{(j)}-\tau_{i \mu}^{(j)}\right),
$$

where $\left\{\tau_{i 1}^{(j)}, \ldots, \tau_{i j}^{(j)}\right\}$ is the increasing rearrangement of $\left\{\tau_{i 1}, \ldots, \tau_{i j}\right\}$. We set

$$
\sigma_{i s}:=\min _{1 \leq j \leq l} \sigma_{i j s} .
$$

We define

$$
\Delta_{m}:=\max _{l(m) \leq i \leq r(m)-1}\left(x_{i+1}-x_{i}\right),
$$

so that the norm of $\Pi_{n}$ has the form

$$
H_{n}:=\max _{p \leq m \leq n} \Delta_{m},
$$

and we define

$$
\tilde{H}_{n}:=\min _{p \leq m \leq n} \Delta_{m} .
$$

Moreover, we set

$$
\begin{aligned}
\delta_{m} & :=\min _{\substack{l(m) \leq i \leq r(m)-1 \\
i: x_{i}<x_{i+1}}}\left(x_{i+1}-x_{i}\right), \\
\delta_{m, p-r} & :=\min _{m+1-p+r \leq \nu \leq m}\left(x_{\nu+p-r}-x_{\nu}\right), \quad r=0,1, \ldots, p-1 .
\end{aligned}
$$

The following lemma provides a bound for $\left|\lambda_{i j} R\right|$, when $R$ is defined as in (14). 
Lemma 4. Let $1 \leq s \leq l$. Let $m+1-p \leq i \leq m$. Then, if $f \in C^{s-1}\left(I_{m}\right)$,

$$
\begin{aligned}
\left|\lambda_{i j} R\right| \leq & (p+[p / 2]-1) \omega\left(D^{s-1} f ; \Delta_{m} ; I_{m}\right) \\
& \cdot \begin{cases}\frac{\left|\eta_{i j}-t\right|^{s-j}}{(j-1) !(s-j) !}, & j=1,2, \ldots, s, \\
\frac{2^{j-s}}{(s-1) ! \sigma_{i j, j-1} \cdots \sigma_{i j s}}, & j=s+1, \ldots, l,\end{cases}
\end{aligned}
$$

where $\eta_{i j} \in I_{i j}$.

Proof. The proof is the same as in [6, Lemma 5.1] if one takes in account that, for any $x \in I_{m}$ and $t \in J_{m}$, we have

$$
\left|D^{s-1} f(x)-D^{s-1} f(t)\right| \leq(p+[p / 2]-1) \omega\left(D^{s-1} f ; \Delta_{m} ; I_{m}\right) .
$$

Now, we can state the following theorem, which provides a local error estimate.

Theorem 4. If $f \in C^{s-1}\left(I_{m}\right)$ with $1 \leq s \leq l \leq p$, then for $0 \leq r<p$,

$$
\max _{t \in J_{m}}\left|E_{r s}^{n}(t)\right| \leq C_{m} \Delta_{m}^{s-r-1} \omega\left(D^{s-1} f ; \Delta_{m} ; I_{m}\right),
$$

where

$$
\begin{aligned}
C_{m}:= & p(p+[p / 2]-1)[p+2([p / 2]-1)]^{s-1} \Gamma_{p r}\left(\frac{\Delta_{m}}{\delta_{m, p-r}}\right)^{r} \\
& {\left[\sum_{\substack{j=1 \\
j \neq 2}}^{s} \frac{1}{(j-1) !(s-j) !}+\frac{1}{(s-1) !} \cdot \sum_{\substack{j=s+1 \\
j \neq 2}}^{l}\left(2 \rho_{m}\right)^{j-s}\right] }
\end{aligned}
$$

and

$$
\rho_{m}:=\max _{m+1-p \leq i \leq m} \frac{x_{\bar{r}(i)}-x_{\bar{l}(i)}}{\sigma_{i s}} .
$$

Proof. We sketch the proof, which is similar to the proof in [6, Theorem 5.2].

Since $\eta_{i j} \in I_{i j} \subset I_{m}$, by (11), (12) and (13) we have

$$
\left|\eta_{i j}-t\right| \leq(p+[p / 2]-1) \Delta_{m}
$$

Since both $x_{\nu_{\mu}}$ and $\tau_{i \mu}$ belong to $I_{i j}$, then, from (8), (9) and (10),

$$
\left|x_{\nu_{\mu}}-\tau_{i \mu}\right| \leq\left|x_{\bar{r}(i)}-x_{\bar{l}(i)}\right| \leq[p+2([p / 2]-1)] \Delta_{m}
$$

holds. 
By (17), (18), (22) and (23) we can write

$$
\begin{aligned}
& \left|E_{r s}^{n}(t)\right| \leq p(p+[p / 2]-1) \Gamma_{p r} \max _{m+1-p \leq i \leq m}\left[\sum_{\substack{j=1 \\
j \neq 2}}^{s} \frac{\left|\eta_{i j}-t\right|^{s-j}}{(j-1) !(s-j) !} A_{i j}\right. \\
& \left.+\sum_{\substack{j=s+1 \\
j \neq 2}}^{l} \frac{2^{j-s}}{(s-1) ! \sigma_{i j, j-1} \cdots \sigma_{i j s}} A_{i j}\right] \omega\left(D^{s-1} f ; \Delta_{m} ; I_{m}\right) \\
& \leq p(p+[p / 2]-1) \Gamma_{p r} \\
& \cdot\left\{\sum_{\substack{j=1 \\
j \neq 2}}^{s} \frac{\left[(p+2([p / 2]-1)) \Delta_{m}\right]^{s-j}}{(j-1) !(s-j) !} \cdot \frac{\left[(p+2([p / 2]-1)) \Delta_{m}\right]^{j-1}}{\delta_{m, p-r}^{r}}\right. \\
& \left.+\sum_{\substack{j=s+1 \\
j \neq 2}}^{l} \max _{m+1-p \leq i \leq m}\left[\frac{2^{j-s}}{(s-1) ! \sigma_{i s}^{j-s}} \frac{\left(x_{\bar{r}(i)}-x_{\bar{l}(i)}\right)^{j-1}}{\delta_{m, p-r}^{r}}\right]\right\} \omega\left(D^{s-1} f ; \Delta_{m} ; I_{m}\right) .
\end{aligned}
$$

Formulas (19), (20) and (21) follow from (24).

We now state the following corollary:

Corollary 5. Let $f \in C^{s-1}\left(I_{m}\right)$ with $1 \leq s \leq l \leq p$, and assume that $Q_{n} f$ belongs to a LU spline space $\mathbf{S}_{p, \pi_{n}}$ with constant $A$. Then, for $0 \leq r<p$, there holds

$$
\max _{t \in J_{m}}\left|E_{r s}^{n}(t)\right| \leq \bar{C}_{r} \Delta_{m}^{s-r-1} \cdot \omega\left(D^{s-1} f ; \Delta_{m} ; I_{m}\right) .
$$

Proof. By Theorem 4 we need only prove that $\rho_{m}$ and $\Delta_{m} / \delta_{m, p-r}$ are uniformly bounded for all $m$ and $n$.

Since, from (12) and (13), one has $r(m)-l(m) \leq 2(p+[p / 2])-3$, by local uniformity, we have for all $m+p-1 \leq i \leq m$,

$$
x_{\bar{r}(i)}-x_{\bar{l}(i)}<x_{r(m)}-x_{l(m)} \leq \delta_{m} \sum_{k=0}^{2(p+[p / 2])-4} A^{k} .
$$

Assuming that $\tau_{i \mu}^{(l)}=\zeta_{\nu}, 1 \leq \mu \leq l-1$, we have

$$
\tau_{i, \mu+1}^{(l)}-\tau_{i \mu}^{(l)}=\zeta_{\nu+1}-\zeta_{\nu}=\left(x_{\nu+p}-x_{\nu+1}\right) /(p-1) \geq \delta_{m} /(p-1) .
$$

Inserting (26) and (27) in (21) and recalling $\sigma_{i s} \geq \sigma_{i 1}$, we get

$$
\rho_{m} \leq \rho
$$

with

$$
\rho:=(p-1) \sum_{k=0}^{2(p+[p / 2])-4} A^{k}
$$


Moreover, by using local uniformity, we have

$$
\delta_{m} \geq \Delta_{m} A^{-[2(p+[p / 2])-4]}
$$

and, since $\delta_{m, p-r} \geq \delta_{m}$,

$$
\frac{\Delta_{m}}{\delta_{m, p-r}} \leq A^{2(p+[p / 2])-4}
$$

Inserting (28) and (29) in (20), we obtain the error bound (25).

Note that, from Lemma 2, whenever we write that a result is true for $t \in J_{m}$, then it is true for all relevant $r$ and $s$ if $t \in\left(x_{m}, x_{m+1}\right)$. When $r=0$, it is true for all relevant $s$ if $t \in\left[x_{m}, x_{m+1}\right]$.

Corollary 6. Let $f \in C^{s-1}[a, b]$ with $1 \leq s \leq l \leq p$, and assume that $Q_{n} f$ satisfies the hypothesis of Corollary 5. Then, for $0 \leq r<s$, the uniform bound (6) holds with $C_{r}=\bar{C}_{r}$, and for $s \leq r<p$

$$
\left\|D^{r} Q_{n} f\right\|_{\infty} \leq \bar{C}_{r} \tilde{H}_{n}^{s-r-1} \omega\left(D^{s-1} f ; H_{n} ;[a, b]\right) .
$$

Proof. From Corollary 5 our assertion follows immediately since for all $m$ and $n$

$$
\tilde{H}_{n} \leq \Delta_{m} \leq H_{n}
$$

Finally we state the following lemma:

Lemma 7. Suppose $Q_{n} f$ satisfies the hypothesis of Corollary 5; then the values $v_{i j}$ defined by (4) satisfy

$$
\left|v_{i j}\right| \leq \sum_{\mu=j}^{l} \rho^{\mu-1} .
$$

Proof. From (15), (21) and (28) it follows that

$$
\left|\alpha_{i \mu}\right| \leq\left(\rho \cdot \sigma_{i 1}\right)^{\mu-1} .
$$

Moreover,

$$
\prod_{\substack{s=1 \\ s \neq j}}^{\mu}\left(\tau_{i j}-\tau_{i s}\right) \geq \sigma_{i 1}^{\mu-1}
$$

\section{Application to NUMERICAL INTEGRATION AND COMPUTATIONAL RESUlts}

Convergence results were already proved for product quadrature rules based on QI splines using parameters $T_{1}-T_{5}$ in $[1,3-5]$. These convergence results are both for bounded $[1,3,4]$ and unbounded integrands [4]. Pointwise and uniform convergence results are proved in $[1,3,5]$ for sequences of CPV integrals of these splines. We apply these convergence results to the QI splines based on $T_{6}$. 
From Corollary 6 with $s=1, r=0$ and from Lemma 7, it can be proved as in [1] that

$$
\mathcal{I}\left(K Q_{n} f\right):=\int_{-1}^{1} K(x) Q_{n} f(x) d x \rightarrow \mathcal{I}(K f) \text { as } n \rightarrow \infty
$$

for $K \in \mathbf{L}_{1}(J)$, with $J:=[-1,1]$ and $f \in \mathbf{R}(J)$, the set of all (bounded) Riemannintegrable functions.

By Corollary 5 with $s=1, r=0,1$, it can be proved as in [1] that

$$
\mathcal{J}\left(u Q_{n} f ; \lambda\right):=f_{-1}^{1} u(x) \frac{Q_{n} f(x)}{x-\lambda} d x \rightarrow \mathcal{J}(u f ; \lambda) \quad \text { as } \quad n \rightarrow \infty
$$

where $f_{-1}^{1}:=\lim _{\varepsilon \rightarrow 0}\left\{\int_{-1}^{\lambda-\varepsilon}+\int_{\lambda+\varepsilon}^{1}\right\}$, for $u$ and $f$ such that $\mathcal{J}(u f ; \lambda)$ exists and $\lambda \in \stackrel{\circ}{J}:=(-1,1)$.

Introducing the modified QI splines

$$
\hat{Q}_{n} f(x)=f(-1) N_{1 n}^{p}(x)+\sum_{i=2}^{n-1} N_{i n}^{p}(x) \sum_{j=1}^{l} v_{i j} f\left(\tau_{i j}\right)+f(1) N_{n n}^{p}(x),
$$

which can be defined using parameters $T_{6}$ since $\tau_{11}:=\zeta_{1}=-1$ and $\tau_{1 n}:=\zeta_{n}=1$, we can prove as in [5] that

$$
\mathcal{J}\left(w \hat{Q}_{n} f ; \lambda\right) \rightarrow \mathcal{J}(w f ; \lambda) \quad \text { as } n \rightarrow \infty,
$$

uniformly with respect to $\lambda$ in $\stackrel{\circ}{J}$, for $f \in \mathbf{H}_{\mu}(J)$ with $0<\mu \leq 1$ and $w(x):=$ $(1-x)^{\alpha}(1+x)^{\beta}$ with $\alpha, \beta>-1$, if $\mu+\min (\alpha, \beta)>0$. Here,

$$
\mathbf{H}_{\mu}(J):=\left\{g: \omega(g ; t ; J) \leq B t^{\mu}, B>0,0<\mu \leq 1\right\} .
$$

Finally, we give some computational results obtained by using the quadrature rule

$$
\mathcal{I}\left(K Q_{n} f\right):=\sum_{i=1}^{n} \sum_{j=1}^{p} v_{i j} w_{i n}(K) f\left(\tau_{i j}\right)
$$

where $w_{i n}(K):=\mathcal{I}\left(K N_{i n}^{p}\right)$, to approximate $\mathcal{I}(K f)$ for test functions $f \in \mathbf{R}(J)$ and $K(x):=\ln |x-\lambda|, \lambda \in \stackrel{\circ}{J}$. We define the truncation error of the rule (31) by

$$
R^{(n)}(K f):=\mathcal{I}(K f)-\mathcal{I}\left(K Q_{n} f\right) .
$$

We base our calculations on the algorithm explained in [2], which we generalize slightly by introducing knot sequences with a multiple interior knot. For the test 
function $f(x):=x^{4}+|x|$, which has a singular point of the derivative at $x=0$, we consider the following LU partition of $J$ :

$$
L:\left\{\begin{array}{l}
y_{\nu}:=0, \\
y_{\nu \pm i}:=y_{\nu \pm(i-1)} \pm(i+1) \delta / 2, \quad i=1, \ldots, \nu-1, \\
y_{\nu \pm \nu}:= \pm 1,
\end{array}\right.
$$

where $\delta>0$ and $\nu$ is such that

$$
y_{\nu+(\nu-1)}+(\nu+1) \delta / 2 \geq 1-(\nu+1) \delta / 2 .
$$

We denote by

$R_{L}^{(n)}$ the truncation error (32) of the rule (31) based on the QI points $T_{6}$ and the sequence of knots obtained by giving multiplicity $(p-1)$ to $y_{\nu}=0$ in (33).

$R_{U}^{(n)}$ the trunction error (32) of the rule (31) based on $T_{6}$ and the sequence of simple interior knots obtained from the uniform partition $U$ of $J$, where

$$
U: y_{i}=-1+2 i /(N+1), \quad i=0,1, \ldots, N+1 .
$$

Table 1 reports the absolute errors for different $\delta, n$ and $p$.

TABLE 1. Absolute errors for $K(x):=\ln \left|x-\frac{e}{4}\right|, f(x):=$ $x^{4}+|x|, \mathcal{I}(K f)=-1.14788951532$

\begin{tabular}{rrrrr}
\hline \multicolumn{1}{c}{$p$} & \multicolumn{1}{c}{$\delta$} & $n$ & $\left|R_{L}^{(n)}(K f)\right|$ & $\left|R_{U}^{(n)}(K f)\right|$ \\
\hline 3 & 0.5 & 5 & $4.92(-2)$ & $4.72(-2)$ \\
& 0.05 & 17 & $3.14(-3)$ & $1.25(-3)$ \\
& 0.005 & 55 & $3.48(-5)$ & $9.27(-5)$ \\
& 0.001 & 125 & $1.06(-6)$ & $1.71(-5)$ \\
\hline \multirow{2}{*}{4} & 0.5 & 7 & $1.19(-1)$ & $3.26(-2)$ \\
& 0.05 & 19 & $1.55(-3)$ & $1.26(-3)$ \\
& 0.005 & 57 & $1.53(-5)$ & $9.04(-5)$ \\
& 0.001 & 127 & $5.14(-7)$ & $1.68(-5)$ \\
\hline 5 & 0.5 & 9 & $3.14(-2)$ & $1.25(-2)$ \\
& 0.05 & 21 & $1.67(-4)$ & $8.86(-4)$ \\
& 0.005 & 59 & $1.67(-6)$ & $8.51(-5)$ \\
& 0.001 & 129 & $6.67(-8)$ & $1.65(-5)$ \\
\hline
\end{tabular}

The numerical results in Table 1 show that the partition $L$, with a multiple knot at the singular point of $f^{\prime}(x)$, performs better when $n$ becomes large.

For the test functions $f(x):=x^{4}-\operatorname{sign}(x)$, where $\operatorname{sign}(x):=1$ if $x>0, \operatorname{sign}(0):=$ 0 , and $\operatorname{sign}(x):=-1$ if $x<0$, and for $f(x):=x^{4}+x|x|$, we use the uniform partition $U$ defined by (34) with simple interior knots. We compare in Tables 2 and 3 the absolute errors of the quadrature (31) based respectively on $T_{1}$, with $l=p$, and $T_{6}$ for different $p$ and $n$. We denote by

$R_{T_{1}}^{(n)}$ the truncation error (32) of the rule (31) based on $T_{1}$

$R_{T_{6}}^{(n)}$ the truncation error (32) of the rule (31) based on $T_{6}$. 
TABLE 2. Absolute errors for $K(x):=\ln \left|x-\frac{e}{4}\right|, f(x):=$ $x^{4}-\operatorname{sign}(x), \mathcal{I}(K f)=-1.45785292443$

\begin{tabular}{rrrr}
\hline \multicolumn{1}{c}{$p$} & $n$ & $\left|R_{T_{1}}^{(n)}(K f)\right|$ & $\left|R_{T_{6}}^{(n)}(K f)\right|$ \\
\hline 3 & 4 & $8.96(-1)$ & $1.12(-1)$ \\
& 10 & $6.23(-3)$ & $8.74(-3)$ \\
& 34 & $6.41(-4)$ & $4.88(-4)$ \\
& 130 & $3.99(-5)$ & $3.00(-5)$ \\
\hline \multirow{2}{*}{4} & 5 & $3.99(-1)$ & $9.81(-2)$ \\
& 11 & $6.86(-2)$ & $1.01(-2)$ \\
& 35 & $2.28(-3)$ & $9.37(-4)$ \\
& 131 & $1.35(-4)$ & $5.98(-5)$ \\
\hline 5 & 6 & $7.05(-2)$ & $1.24(-2)$ \\
& 12 & $5.45(-2)$ & $3.75(-3)$ \\
& 36 & $9.92(-4)$ & $4.79(-4)$ \\
& 132 & $6.12(-5)$ & $2.99(-5)$ \\
\hline
\end{tabular}

TABLE 3. Absolute errors for $K(x):=\ln \left|x-\frac{e}{4}\right|, f(x):=$ $x^{4}+x|x|, \mathcal{I}(K f)=-1.09751837560$

\begin{tabular}{rrrr}
\hline \multicolumn{1}{c}{$p$} & $n$ & $\left|R_{T_{1}}^{(n)}(K f)\right|$ & $\left|R_{T_{6}}^{(n)}(K f)\right|$ \\
\hline \multirow{2}{*}{3} & 4 & $5.91(-1)$ & $8.30(-2)$ \\
& 10 & $8.57(-3)$ & $3.53(-4)$ \\
& 34 & $9.71(-6)$ & $3.93(-6)$ \\
& 130 & $2.03(-8)$ & $2.13(-8)$ \\
\hline \multirow{2}{*}{4} & 5 & $9.07(-2)$ & $4.78(-2)$ \\
& 11 & $8.61(-3)$ & $1.84(-3)$ \\
& 35 & $6.57(-5)$ & $1.22(-5)$ \\
& 131 & $2.69(-7)$ & $5.00(-8)$ \\
\hline \multirow{2}{*}{5} & 6 & $2.23(-2)$ & $7.54(-3)$ \\
& 12 & $1.77(-4)$ & $2.10(-4)$ \\
& 36 & $7.12(-8)$ & $3.40(-7)$ \\
& 132 & $2.12(-10)$ & $1.29(-9)$ \\
\hline
\end{tabular}

The numerical results of Tables 2 and 3 confirm that the approximations, based on the QI points $T_{6}$ (that need only $n$ function evaluations ), have the same performance as the ones based on $T_{1}$ (that need up to $n(p-1)-p+2$ function evaluations). 


\section{REFERENCES}

1. C. Dagnino, V. Demichelis, E. Santi, Numerical integration based on quasi-interpolating splines, Computing 50 (1993), 149-163. MR 94i:65028

2. __ An algorithm for numerical integration based on quasi-interpolating splines, Numerical Algorithms 5 (1993), 443-452. MR 94k:65029

3. _ Local spline approximation methods for singular product integration, to appear in Approximation Theory and its Applications.

4. C. Dagnino and P. Rabinowitz, Product integration of singular integrands using quasiinterpolatory splines, to appear in Intern. J. Comput. Math. special issue dedicated to 100th birthday of Cornelius Lanczos.

5. V. Demichelis, Uniform convergence for Cauchy principal value integrals of modified quasiinterpolatory splines, Intern. J. Comput. Math. 53 (1994), 189-196.

6. T. Lyche and L. L. Schumaker, Local spline approximation methods, J. Approx. Theory $\mathbf{1 5}$ (1975), 294-325. MR 53:1108

7. L. L. Schumaker, Spline functions, New York: John Wiley \& Sons, 1981. MR 82j:41001

Department of Mathematics, University of Torino, Via Carlo Alberto 10, I-10123, TORINO, ITALY

E-mail address: demichelis@dm.unito.it 